\title{
RU486 Reverses Emotional Disorders by Influencing Astrocytes and Endoplasmic Reticulum Stress in Chronic Restraint Stress Challenged Rats
}

\author{
Liru Donga,b Songjun Wanga Yingmin Lia Ziqin Zhaoc Yiwen Shen ${ }^{c}$ Li Liu ${ }^{d}$ \\ Guangming $\mathrm{Xu}^{\mathrm{a}}$ Chunling $\mathrm{Ma}^{\mathrm{a}}$ Shujin Lia Xiaojing Zhang ${ }^{\mathrm{a}}$ Bin Cong ${ }^{\mathrm{a}}$ \\ ${ }^{a}$ Department of Forensic Medicine, Basic Medical College, Hebei Medical University, Hebei Key \\ Laboratory of Forensic Medicine, Shijiazhuang, Hebei, bDepartment of Pathology, Affiliated Hospital of \\ North China University of Science and Technology, Tangshan, Hebei, 'Department of Forensic Medcine, \\ School of Basic Medical Sciences, Fudan University,Shanghai, dForensic Science Service of the Beijing \\ Public Security Bureau, Beijing, China
}

\section{Key Words}

Chronic • Restraint Stress • Astrocyte - Apoptosis • RU486 • Endoplasmic Reticulum (ER) Stress

\begin{abstract}
Aims: To investigate the effect of RU486 (mifepristone) on emotional disorders in chronic restraint stress-induced rats and to explore the mechanisms of that phenomenon. Methods: For this purpose, 80 healthy male Sprague Dawley rats were randomly divided into four groups: the normal group (Con group, The Con group members received no treatment, eating and drinking freely), the chronic restraint stress group (CRS group, normal Sprague Dawley rats treated with chronic restraint stress, $6 \mathrm{~h}$ /day for 21days), the propylene glycol group (CRS+ propylene glycol) and the RU486 group (CRS+RU486). RU486 or propylene glycol was administered 30 mins before each CRS procedure. Twenty-four hours after CRS exposure, we investigated the effects of CRS on the anxiety-like behavior using an elevated plus-maze (EPM). To explore the mechanisms of RU486 on anxiety, we measured the expression of glial fibrillary acid protein (GFAP) and $\beta$-subunit of S100 (S100 $\beta$ ) via immunohistochemistry and western blot analysis. Apoptosis was demonstrated by flow cytometry. In addition, endoplasmic reticulum (ER) stress markers, glucose regulated protein 78 (GRP78), C/EBP homologous protein (CHOP) and Cysteine aspartic acid specific protease-12 (Caspase-12), were detected by western blot analysis. Results: Compared to the control group, rats in the CRS and propylene glycol group showed decreased exploratory behavior on the open arms during EPM testing, and these reductions were accompanied by significantly reduced GFAP and S100 $\beta$ expression, increased apoptosis and GRP78, CHOP, and caspase-12 expression in the amygdala. However, RU486 increases the exploratory behavior and reverses the changes of GFAP, S100ß, GRP78, CHOP, and caspase-12 and protects cells against apoptosis. Conclusions: Taken together, these data

Bin Cong, MD, PhD,

and Xiaojing Zhang, MD, PhD


suggest that exposure to chronic restraint stress decreases the number of astrocytes and induces apoptosis and ER stress in the amygdala, which are possible causes for psychiatric disorders. RU486 can significantly ameliorate abnormal behaviors in CRS-induced anxiety model rats. The protective effects of RU486 could be attributed to its anti-ER stress, antiapoptosis and astrocyte increasing effects.

\section{Introduction}

Stress is known to be a major risk factor in the etiology of several psychiatric diseases, such as anxiety and depression, which occur because of increased glucocorticoid neurotoxicity. Adaptation to stress is critical for maintaining physical and mental health. However, when stressors are overwhelming or are repeated over time they can eventually lead to mental illness $[1,2]$, and the related hypersecretion of glucocorticoids are associated with psychiatric disorders ranging from hyper emotional states to mood dysfunction [2, 3]. The amygdala is involved in the emotional value of mood and cognition, and plays a critical role in fear and anxiety-related behaviors. Several studies have revealed the tight association of CRS-related mental disorders with changes in dendritic spine number and dendritic length, as these may exert significant functional effects on fear behavior [4, 5]. Neuroimaging studies investigating major depression in patients and postmortem with reveal volumetric and functional changes of the amygdala [6, 7].

Glial cells constitute approximately $50 \%$ of total brain cells [8]. Abundant glucocorticoid receptors are present in cytoplasm of both neurons and astrocytes of the amygdala, and amygdala activity and volumetric changes have been ascribed to both neurons and glial cells [9]. Glial cells are known to play an important role in the normal functioning of the nervous system, but there are no studies evaluating the effects of astrocytes in the amygdala on emotional symptoms. Moreover, postmortem studies report reduced glial densities in the amygdala of depressed patients [7]. Astrocyte apoptosis is considered a critical factor in the development of several central nervous system injuries and degenerative diseases [10]. Several studies have demonstrated that increased ER stress participates in astrocyte apoptosis [11]. Stress induces psychiatric diseases in vivo, and corticosterone treatment remarkably increases the percentage of apoptotic astrocytes through ER stress in vitro [12]. Therefore, ER stress may play an important role in the development of CRS-related astrocytic functions.

RU486 is effective against the deleterious effects of excess glucocorticoid production, and ameliorates the effects of elevated glucocorticoids as a result of Cushing's syndrome. Moreover, several studies have demonstrated that RU486 targeting the glucocorticoid receptor may ameliorate stress dysfunction [13], but the potential mechanism by which RU486 acts as a protective agent is incompletely understood. Hence, drugs targeting glucocorticoid receptors may help to advance discovery of strategies to reduce the burden of stress-related neuropsychiatric disorders.

Understanding how the RU486 is associated with behavior deficits, apoptosis, ER stress, and glial loss is critical for the development of effective treatment strategies. Thus, we conducted this study to observe the effects of RU486 in CRS-induced anxiety-like rats, and explore the mechanisms of RU486 on chronic stress.

\section{Materials and Methods}

\section{Animals}

All experimental procedures for animal experiments performed in this study were conducted in accordance with the National Institutes of Health Guide for the Care and Use of Laboratory Animals. The animal protocol was approved by the Local Committee of Animal Use and Protection of Hebei Medical 


\section{Cellular Physiology Cell Physiol Biochem 2017;42:1098-1108 \begin{tabular}{ll|l} 
and Biochemistry Published onlıne: June 29, 2017 & $\begin{array}{l}\text { C) } 2017 \text { The Author(s). Published by S. Karger AG, Basel } \\
\text { www.karger.com/cpb }\end{array}$ \\
\hline
\end{tabular}}

Dong et al.: RU486 and Chronic Restraint Stress

University. Male Sprague-Dawley rats (6-8 weeks old, weighing 200-250 g; the Center of Laboratory Animal Science, Hebei Medical University, China.) were housed two per cages under a 12:12 h light-dark schedule (lights on at 07:30 to19:30), with food and water being freely available. Constant temperature $\left(23 \pm 1^{\circ} \mathrm{C}\right)$ and humidity $(60 \pm 10 \%)$ were maintained throughout the experiments.

Reagents

RU486 (mifepristone; Sigma-Aldrich), a glucocorticoid receptor antagonist, was dissolved in $100 \%$ propylene glycol.

Stress protocol

Eighty healthy male Sprague Dawley rats were randomly divided into four groups: the normal group (Con group, The Con group members received no treatments, eating and drinking freely), the chronic restraint stress group (CRS group, normal Sprague Dawley rats treated with chronic restraint stress, 6 h/ day for 21days), the propylene glycol group (CRS+ propylene glycol) and the RU486 group (CRS+RU486). Rats in CRS, Propylene glycol, and RU486 groups were subjected to CRS $6 \mathrm{~h}$ /day for 21 days. The restraint period occurred during the light cycle, randomly selected from 07:30 am to 7:30 pm to prevent adaptation to a fixed binding time. Rats were bound to a type T binding platform, which consists of the base $(10 \mathrm{~cm} \times 20$ $\mathrm{cm} \times 2.8 \mathrm{~cm})$ and the upper part of the binding platform $(22 \mathrm{~cm} \times 6.6 \mathrm{~cm})$. The upper part had two small frames for fixing the head and four small grooves for fixing the limbs, and had two adjustable soft belts for fixing the abdomen and chest, respectively [14]. The animals fit tightly on the T binding platform, and it was impossible for them to move or turn around. To observe the effects of RU486 $(10 \mathrm{mg} / \mathrm{kg}$, intraperitoneal, Sigma-Aldrich, USA) on CRS rats, RU486 or propylene glycol was administered 30 mins before each restraint stress procedure. Before sacrifice, all rats were prepared for behavior tests.

Elevated plus maze (EPM) testing for anxiety and spontaneous locomotor activity measurement

To verify the effectiveness of emotion changes, we tested animals in the EPM. One day after the final restraint/control handling session. The maze consisted of two open arms $(50 \times 15 \mathrm{~cm})$ and two closed arms $(50 \times 15 \times 40 \mathrm{~cm})$. The apparatus was placed at a height of $50 \mathrm{~cm}$ above the ground. All sides and bottom surfaces of the opened and closed arms were constructed from black Plexiglas. At the start, rats were individually placed at the center of the maze facing one open arm and allowed 5 mins of free exploration. The time spent in the open arms as a percentage of the total time spent exploring the EPM was measured during each 5 min test. In addition, the numbers of entries into the open arm as a percentage of the total number of entries was also recorded. The percentages of time spent in open arms and entries into the open arms of total entries were used as an index of anxiety-like behavior. EPM testing was performed once per rat. In addition, the total distance and total arm entries traveled by rats were recorded as a relative index of spontaneous locomotor activity.

\section{Western blot analysis}

Rats were sacrificed after EPM testing. Brains were immediately removed on ice. Amygdala tissue from each group were dissected and homogenized in RIPA buffer containing Halt protease and phosphatase inhibitor cocktail (Sigma-Aldrich) and lysates were centrifuged at $4^{\circ} \mathrm{C}(14,000 \mathrm{xg})$ for $10 \mathrm{mins}$ to pellet and eliminate cellular debris. Supernatants were then stored at $-80^{\circ} \mathrm{C}$ until use. Protein concentrations were calculated using a BCA Protein Assay Kit (Solarbio, Beijing, China). Twenty micrograms of total protein from each sample were separated in 10.5\% SDS-PAGE gel in a Bio-Rad mini tank apparatus and transferred to PVDF membranes by a Bio-Rad Trans-blot turbo transfer system. Membranes were then blocked with $5 \%$ nonfat dry milk for $1 \mathrm{~h}$ at room temperature, followed by primary incubation with anti-GFAP (1:1000, Abcam), anti-S100ß (1:500,Abcam), anti-GRP78 (1:500,santa Cruz), anti-CHOP (1:1000,cell signaling), anticaspase-12 (1:1000, cell signaling), and GAPDH (1:1000, Santa Cruz) antibodies for $1 \mathrm{~h}$ at room temperature, then $4^{\circ} \mathrm{C}$ for $24 \mathrm{~h}$. Immuno-reactive bands were then probed with IgG (1:5000, Santa Cruz) for $1 \mathrm{~h}$ and developed using an ECL detection system (Thermo Fisher Scientific, Rockford, IL, USA). Optical densities of immune-reactive bands were quantified using NIH Image J software, and the results were expressed as a ratio of proteins of interest and GAPDH. 


\section{Cellular Physiology Cell Physiol Biochem 2017;42:1098-1108 \begin{tabular}{lll} 
DOI: 10.1159/000478764 & and Biochemistry & $\begin{array}{l}\text { O 2017 The Author(s). Published by S. Karger AG, Basel } \\
\text { www.karger.com/cpb }\end{array}$ \\
\cline { 2 - 3 }
\end{tabular} ang et al:: RU486 and Chronic Restraint Stress}

Immunohistochemistry

Rats were deeply anesthetized with an intraperitoneal injection of pentobarbital sodium (50 mg/kg) and transcardially perfused with $100 \mathrm{ml}$ phosphate-buffered saline (PBS, pH=7.4) followed by $100 \mathrm{ml} 4 \%$ paraformaldehyde in PBS. Brains were dissected and post-fixed in the same fixative overnight at $4^{\circ} \mathrm{C}$, then dehydrated and embedded in paraffin. Next, 5 - $\mu$ m-thick sections were cut serially (brain sections from -1.80 $\mathrm{mm}$ to $-3.30 \mathrm{~mm}$ posterior to bregma were analyzed). Five slides were randomly selected from each group, and in each slide five visual fields $(\times 400)$ were randomly selected in the amygdala. The number of positive cells and total cells were counted in each slide. The percentage of positive cells $=$ (the number of positive cells/total cells) $\times 100 \%$. Sections were mounted on Menzel Plus slides and used for immunohistochemistry.

For immunohistochemical experiments, sections were deparaffinized, rehydrated and washed in PBS before heating in a microwave oven containing citrate buffer (PH6.0, 0.01 M) for antigen retrieval. The sections were heated for $10 \mathrm{~min}$ followed by a cooling-down period of $2 \mathrm{~h}$. The sections were washed in PBS and treated with $0.5 \%$ hydrogen peroxide $\left(\mathrm{H}_{2} \mathrm{O}_{2}\right)$ in PBS, washed three times in PBS, and preincubated in normal goat serum for $1 \mathrm{~h}$ at $37^{\circ} \mathrm{C}$, and incubated with primary antibodies (GFAP 1:1000, S-100ß1:50, Abcam) at $4^{\circ} \mathrm{C}$ for $24 \mathrm{~h}$. Sections were washed six times in PBS and incubated with biotinylated goat-anti rabbit/mouse IgG for $30 \mathrm{~min}$ at $37^{\circ} \mathrm{C}$, washed three times in PBS, and processed by using the horseradish peroxidase-Streptavidin (Universal Streptavidin-Peroxidase 9000 kit, Zsbio, China) for 30 min at $37^{\circ} \mathrm{C}$. The sections were washed in PBS and reacted with 3,30-diaminobenzidine tetrahydrochloride. Sections were counter stained with hematoxylin, then dehydrated and cover slipped.

\section{Double-labeled flow cytometry (FCM) analysis}

All rats in four groups were decapitated, and bilateral amygdala were dissected. To prepare monoplast suspensions Each sample was immersed into cold PBS with ophthalmic scissors and snipped into even smaller chunks approximately $1 \mathrm{~mm}^{3}$. Through grinding, sieving and PBS washing single cells were collected, and then adjusted to concentration of $1 \times 10^{6} \mathrm{cells} / \mathrm{ml}$, followed by centrifugation three times to clean and obtain cell pellets. Cell pellets were re-suspended in $200 \mu$ l binding buffer, and then incubated with $5 \mu \mathrm{L}$ Annexin V-FITC and $5 \mu \mathrm{l}$ PI at room temperature for $15 \mathrm{~min}$. Finally, $290 \mu \mathrm{l}$ binding buffer was added, then samples were analyzed by flow cytometry (Becton Dickinson, USA). Flow cytometry pictographs show: viable cell population (Annexin $\mathrm{V}^{-} / \mathrm{PI}^{-}$) in region B3; early apoptotic cell population (Annexin $\mathrm{V}^{+}$/ $\mathrm{PI}^{-}$) in region $\mathrm{B} 4$; late apoptotic and necrotic cell populations (Annexin $\mathrm{V}^{+} / \mathrm{PI}^{+}$) in region $\mathrm{B} 2$; necrotic cell population (Annexin $\mathrm{V}^{-} / \mathrm{PI}^{+}$) in region B1. The total apoptotic rate =the rate of early apoptotic cells(B4) + the rate of late apoptotic and necrotic cells(B2).

\section{Data analysis}

Data are presented as the mean \pm SD and were analyzed by SPSS 17.0 software. Data from all quantitative analyses were subjected to one-way ANOVA followed by LSD post- hoc test for multiple comparisons. $p<0.05$ was considered to be the criterion for statistical significance.

\section{Results}

\section{Effects of CRS and RU486 treatment on behavior}

The CRS rats displayed a significantly decreased percentage of time spent in open arms. However, the RU486 group animals showed a significant increase in the percentage of time spent in open arms compared with the CRS and propylene glycol group animals $\left(\mathrm{F}_{(3,36)}=14.85\right.$, $p<0.0001$, Fig. 1A), but there was no significant difference between the CRS and propylene glycol group animals $(p=0.191)$. Similar changes were seen in percentage of entries into the open arms $\left(\mathrm{F}_{(3,36)}=13.43, p<0.0001\right.$, Fig. 1B), and there was no significant difference between the CRS and propylene glycol group animals $(p=0.828)$. However, there was no significant difference in the total distance $\left(\mathrm{F}_{(3,36)}=0.9570, p=0.4313\right.$, Fig. 1C) and total number of arm entries $\left(\mathrm{F}_{(3,36)}=0.8654, p=0.4679\right.$, Fig. 1D) among the four groups, indicating that CRS has little impact on overall locomotion in rats. Therefore, CRS as a repeated stressor can cause increased anxiety-like behavior, but did not impair locomotor activity. These results indicate that RU486 can reverse the CRS-induced anxiety-like behavior. 
Fig. 1. Effects of CRS and RU486 treatment on anxiety-like behavior. (A) Open arms time / Total time among different groups. (B) Open arms entries / Total entries. (C) Total distance. (D) Total number of arm entries. Statistical analysis was performed using oneway ANOVA. Data were represented as the mean \pm SD. ${ }^{*} p<0.05$, compared with the Con group; $\# p<0.05$, compared with the CRS group.
A

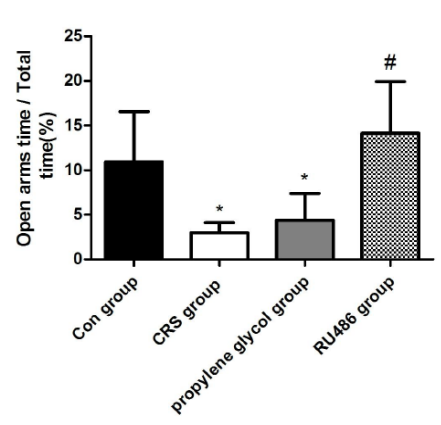

C

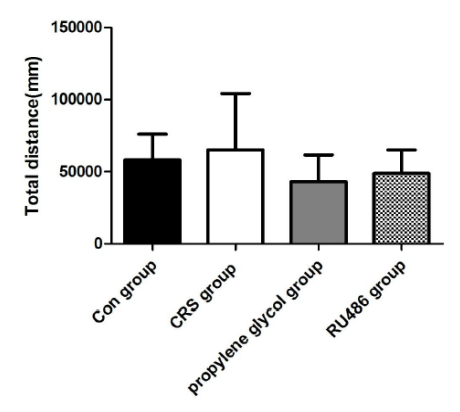

B

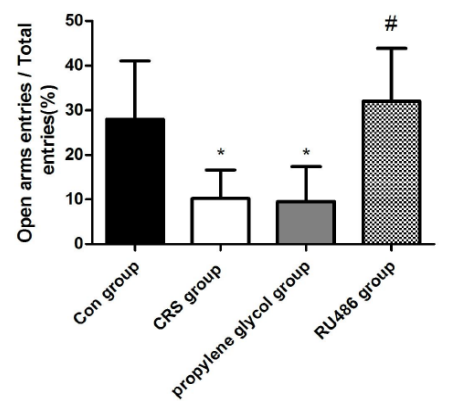

D

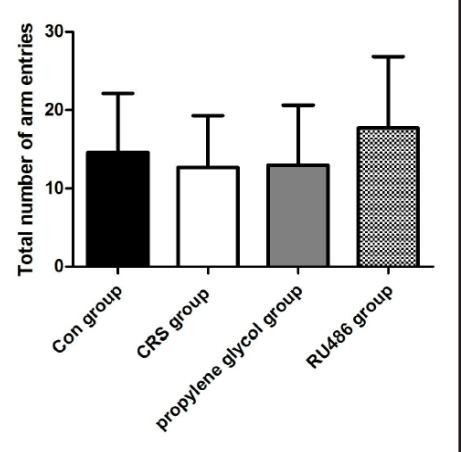

Fig. 2. Effects of CRS and RU486 treatment on GFAP and $\mathrm{S} 100 \beta$ immunoreactivity in the amygdala. (A) Representative images of GFAP and S100 $\beta$ positive cells. (a) GFAP in Con group, (b) GFAP in CRS group, (c) GFAP in propylene glycol group, (d) GFAP in RU486 group, (e) $\mathrm{S} 100 \mathrm{\beta}$ in Con group, (f) $\mathrm{S} 100 \beta$ in CRS group, (g) S100 $\beta$ in propylene glycol group, (h) S100 $\beta$ in RU486 group. (B) Percentages of GFAP and S100 $\beta$ positive cells in 4 groups were calculated. (a) Percentages of GFAP positive cells, (b) Percentages of $S 100 \beta$ positive cells. Five slides were selected from each group, and in each slide,

A

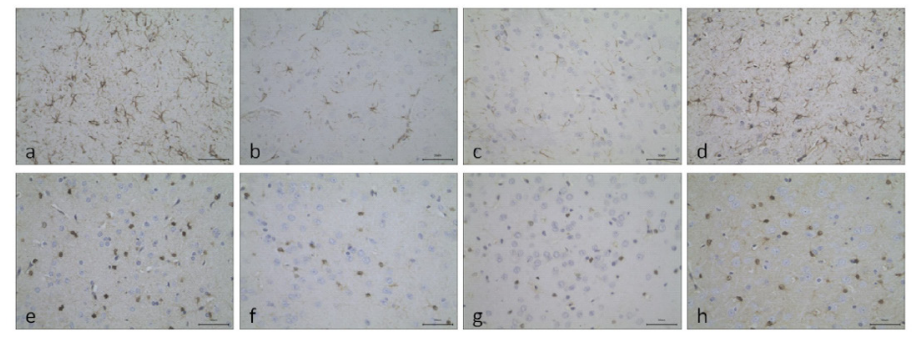

B

a

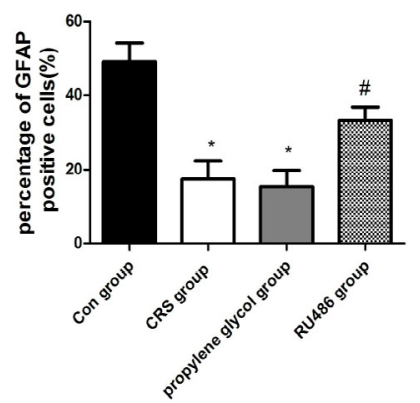

b

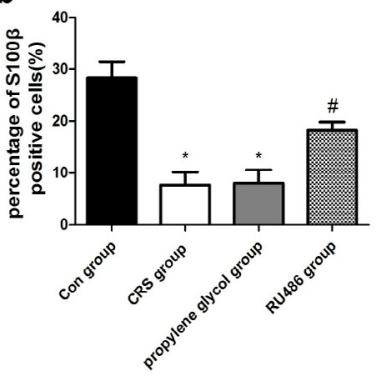

five visual fields ( $\mathrm{x} 400)$ was randomly selected. The numbers of positive cells and total cells were counted in each slide. Scale bar $=50 \mu \mathrm{m}$. The percentage of positive cells=(the number of positive cells/total cells) $\mathrm{x}$ $100 \%$. Statistical analysis was performed using one-way ANOVA. Data were represented as the mean \pm SD. ${ }^{*} \mathrm{p}<0.05$, compared with the Con group; $\# \mathrm{p}<0.05$, compared with the CRS group. 


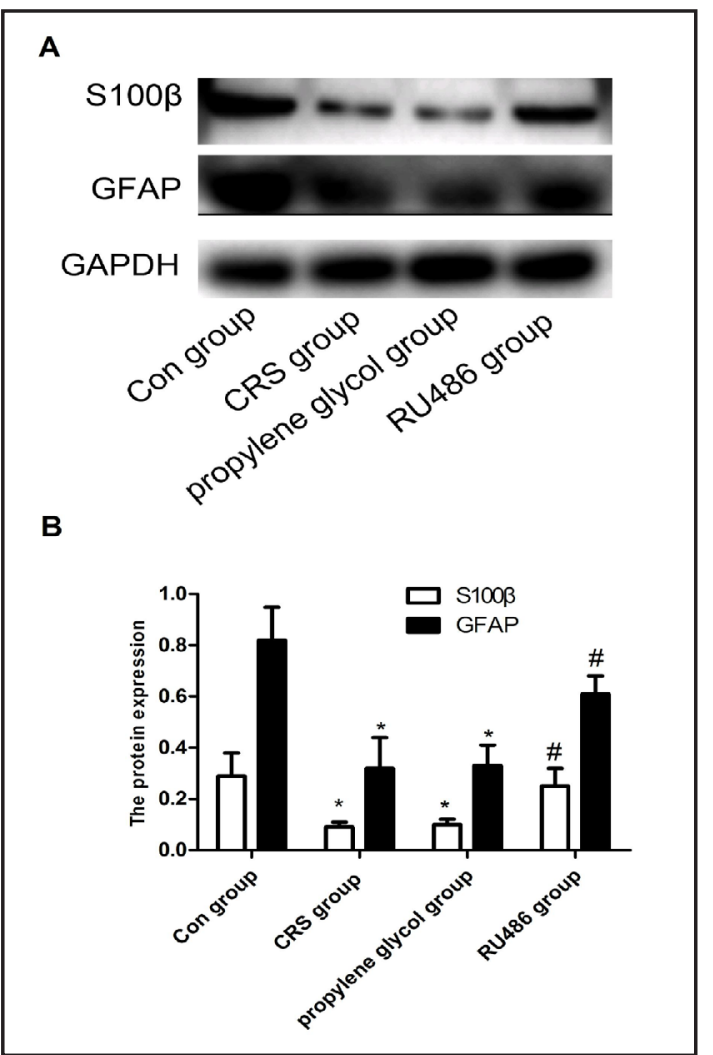

Fig. 3. Changes of GFAP and S100 $\beta$ levels in the amygdala were detected by western blot analysis. GAPDH was used as the internal control, the GFAP protein levels in different groups were expressed as a ratio to that of GAPDH. (A) Representative blots of GFAP and S100 $\beta$. (B) Quantified data of GFAP and S100ß. Statistical analysis was performed using one-way ANOVA. Data were represented as the mean \pm SD. ${ }^{*} p<0.05$, compared with the Con group; $\# p<0.05$, compared with the CRS group.

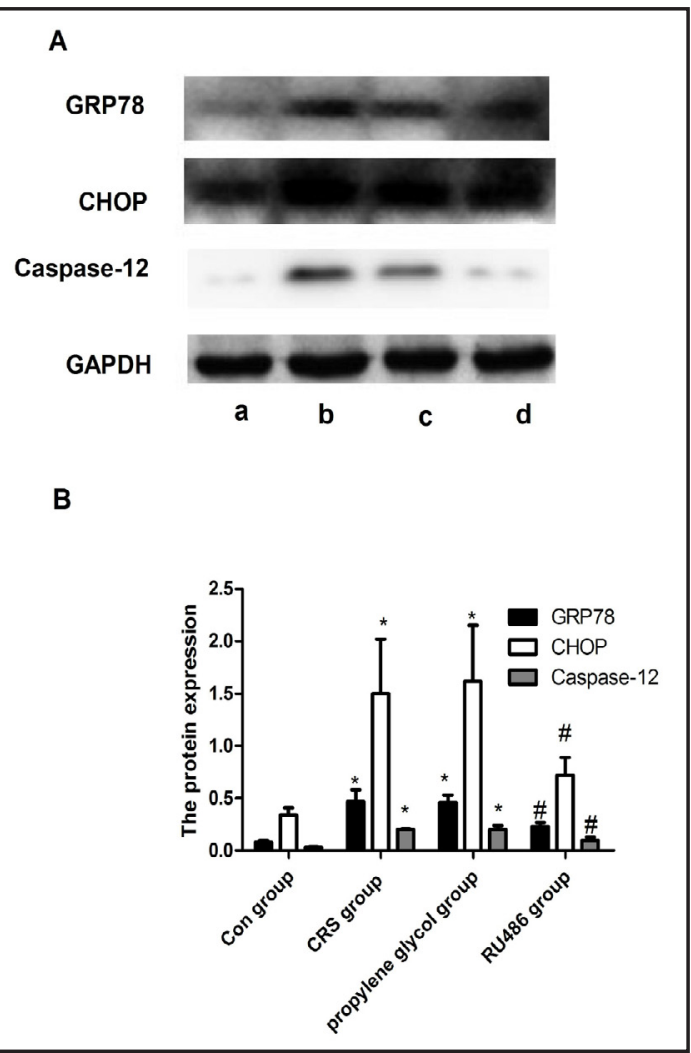

Fig. 4. Changes of GRP78, CHOP and Caspase-12 levels in the amygdala were detected by western blot analysis. GAPDH was used as the internal control; the GRP78, CHOP and Caspase-12 protein levels in different groups were expressed as a ratio to that of GAPDH. (A) Representative blots of GRP78, CHOP and Caspase-12. a: Con group, b: CRS group, c: propylene glycol group, d: RU486 group (B) Quantified data of GRP78, CHOP and Caspase-12. Statistical analysis was performed using one-way ANOVA. Data were represented as the mean $\pm \mathrm{SD}$. ${ }^{*} p<0.05$, compared with the Con group; $\# p<0.05$, compared with the CRS group.

Effects of CRS and RU486 treatment on the expression of GFAP and S100 3 in the amygdala By immunohistochemistry, normal rats showed a moderate immunoreactivity for GFAP and $S 100 \beta$, whereas samples from the CRS and propylene glycol group animals revealed a decreased percentage of immuno-reactive cells compared to normal rats, samples from RU486-treated rats displayed a significantly increased percentage of GFAP and S100 $\beta$ immuno-reactive cells compared to the CRS and propylene glycol animals (GFAP: $\left.\mathrm{F}_{(3,16)}=15.103, p=0.005 ; \mathrm{S} 100 \beta: \mathrm{F}_{(3,16)}=21.128, p=0.002\right)$. However, there is no significant difference between the CRS and propylene glycol group (GFAP: $p=0.635$; S100 $\beta$ : $p=0.882$ ). The results are shown in Fig. 2.

These results were further validated by western blot analysis (see Fig. 3). Statistical results show that GFAP and S100 $\beta$ proteins in the amygdala were significantly decreased in CRS and propylene glycol groups compared to normal rats, but samples from RU486treated rats displayed obviously increased levels of GFAP $\left(\mathrm{F}_{(3,16)}=8.995, p=0.016\right)$ and $\mathrm{S} 100 \beta$ $\left(\mathrm{F}_{(3,16)}=12.991, p=0.007\right)$, compared to the CRS and propylene glycol animals. This finding 


\section{Cellular Physiology Cell Physiol Biochem 2017;42:1098-1108 \begin{tabular}{l|l} 
DOI: 10.1159/000478764 & and Biochemistry 2017 The Author(s). Published by S. Karger AG, Basel \\
wwww.karger.com/cpb
\end{tabular}

Fig. 5. (a) Flow cytometry detected the apoptosis rate in amygdala. Flow cytometry pictographs: viable cell population (Annexin $\mathrm{V}-/ \mathrm{PI}-$ ) in region B3; early apoptotic cell population (Annexin V+/PI-) in region B4; late apoptotic and necrotic cell populations (Annexin $\mathrm{V}+/ \mathrm{PI}+$ ) in region $\mathrm{B} 2$; necrotic cell population (Annexin V-/PI+) in region B1. (A) Con group, (B) CRS group, (C) propylene glycol group, (D) RU486 group. (b) Double-labeled flow cytometry analyzed apoptosis rate in amygdala in 4 groups. The percentages of apoptotic cells remarkably increased in the CRS group, and the increased apoptosis was reduced by RU486; there is no significant difference between the CRS and the propylene glycol group. Statistical analysis was performed using one-way ANOVA. Data were represented as the mean \pm SD. ${ }^{*} p<0.05$, compared with the Con group; \#p<0.05, compared with the CRS group.
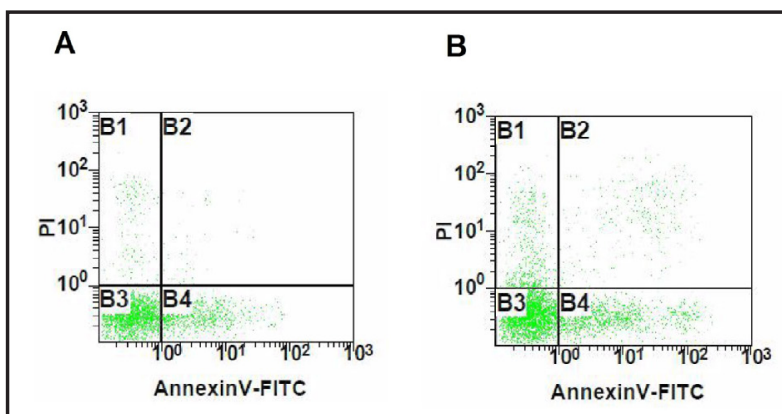

C

$$
\text { D }
$$
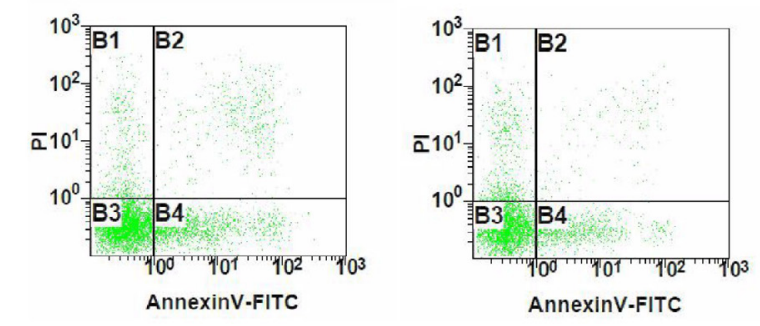

indicates that RU486 can reverse decreases in GFAP and S100 $\beta$ proteins levels. However, there is no significant difference between the CRS and propylene glycol group (GFAP: $p=0.923$; S100ß: $p=0.732$ ).

Effects of CRS and RU486 treatment on expression of GRP78, CHOP and caspase-12 in the amygdala

GRP78 and CHOP can reflect the homeostasis of ER stress. As shown in Fig. 4, western blot analyses demonstrate that CRS significantly increases the expression of GRP78, CHOP and caspase-12 proteins in the amygdala. The GR antagonist RU486 reversed CRS-induced increases in GRP78 $\left(\mathrm{F}_{(3,16)}=4.971, p=0.0351\right)$, $\mathrm{CHOP}\left(\mathrm{F}_{(3,16)}=11.67, p=0.0032\right)$ and caspase-12 $\left(\mathrm{F}_{(3,16)}=11.25, p=0.0036\right)$. However, there is no significant difference between CRS- and propylene glycol groups (GRP78: $p=0.9780$; CHOP: $p=0.4976$; Caspase-12: $p=0.1190$ ). Our results suggest that blocking GRs inhibits CRS-induced ER stress.

\section{Effects of CRS and RU486 treatment on apoptosis}

To examine whether apoptosis was involved in astrocyte injuries and the effects of RU486 on apoptosis, we measured apoptosis via flow cytometry (see Fig. 5). Compared to the con group, the percentage of apoptotic cells were remarkably increased after CRS treatment, and the increase in apoptosis was reduced by RU486 administration. There is no significant difference between the CRS and propylene glycol groups $\left(\mathrm{F}_{(3,16)}=17.131, p=0.000\right)$. Therefore, our data suggest that the blockade of GR activation is responsible for the inhibition of CRSinduced apoptosis.

\section{Discussion}

Stress is one of the major triggers for psychiatric diseases. While the precise mechanism remains unknown, strong evidence has been obtained for glucocorticoid-induced changes in the brain [15]. Several studies have been undertaken to understand impaired glucocorticoid signaling and glucocorticoid associated neurotoxicity $[16,17]$. Physiological or psychological stressors activate the HPA axis, culminating in the biosynthesis and release 
of glucocorticoids from the adrenal cortex. Glucocorticoids exert their biological effects by binding glucocorticoid receptors $[18,19]$. One of the putative mechanisms involved in these changes could be excessive activation of glucocorticoid receptors under stressful conditions [20]. The CRS procedure is a good method to establish the animal anxiety model for analyzing cellular and molecular mechanisms underlying the pathophysiology of mental diseases, and for exploring potential treatments for mood disorders [13]. In the present study, we used the CRS-induced anxiety model as a well-documented animal model of anxiety that results in anxiety-like behavioral effects similar to symptoms observed clinically. The total distance and total arm entries represent the spontaneous movement in the EPM assessment. The percentage of open arms time and total open arms entries during EPM testing were analyzed to evaluate anxiety-like behavior. CRS as a repeated stressor can cause anxiety-like behavior. The CRS group animals showed significant decreases in the percentage of open arms time and total open arm entries, whereas repeated RU486 treatment amended the anxietylike behavior. Ru486 is a glucocorticoid receptor antagonist and a progesterone receptor antagonist. It is widely used in preclinical and clinical treatment for psychotic depression and anxiety. In addition, RU486 alone has no effects on behavior and neuroendocrine function, as confirmed by animal experiments $[13,21-23]$. The present study shows that RU486 can improve the behavioral changes observed in CRS rats.

Astrocytes support and provide protective effects to neurons and are involved in mechanisms of central nervous system injury and degenerative diseases [10, 24]. There is growing evidence that both neuronal and glial changes constitute the neuropathology of neuropsychological disorders $[25,26]$. Thus, changes in astrocytes are likely to play an important role in the etiology of anxiety and mechanism of anxiolytics. An important issue is whether astrocytes can express receptors for hormones (e.g., glucocorticoids) that allow them to directly respond to stress-related chemical mediators. In postmortem human brain, from control subjects, approximately 25\%-30\% of astrocytes express glucocorticoid receptors in the amygdala, whereas this percentage increases to almost $60 \%$ in brains from patients diagnosed with major depression [9]. Glucocorticoid receptors are widely expressed in astrocytes and other glial cells [27], these data are in line with postmortem studies in major depressive disorder [10]. These findings indicate that astrocytes can directly sense and change their morphology or functionality in response to glucocorticoids during stress responses. Thus, these changes of astroglia are likely to have an important functional significance for the etiology of mental illness.

GFAP and S100 $\beta$, markers of astroglia, showed significant decreases in the amygdala of CRS groups compared with the sham group. The level of GFAP expression is linked to reactivity of astrocytes, and because GFAP plays a major role in maintaining the morphology of astrocytes, stress induced decreases in GFAP expression may be due to a rearrangement of astrocytes [28]. Mechanisms involved in the rearrangement of astrocytes possibly include astrocytic death, such as necrosis or apoptosis $[29,30]$. To explore the outcome of the astroglial population, we examined a glia-specific protein called $S 100 \beta$, which is expressed by both protoplasmic and fibrillary astrocytes [31] The results of western blot analysis indicated that reduced expression of these proteins may be due to either: (a) reduction in cell number, or (b) decreased production of GFAP or S100ßproteins without reduction in cell number in the amygdala. However, our immunohistochemistry results indicate a reduced number of $\mathrm{GFAP}^{+}$or $\mathrm{S} 100 \beta^{+}$astrocytes. Together, these studies suggest that the reduced number of GFAP+ or $\mathrm{S} 100 \beta+$ astrocytes within the amygdala may drive the emergence of CRS-related behavioral changes. In addition, RU486 treatment can reverse the CRS-induced decrease in GFAP and S100 $\beta$ expression. In our study, we clearly demonstrate the beneficial effects of RU486 at reversing CRS-induced anxiety-like behaviors. CRS produces an anxietylike behavior and causes a reduced number of $\mathrm{GFAP}^{+}$or $\mathrm{S} 100 \beta^{+}$astrocytes, which are likely to be mediated by glucocorticoid receptor activation.

Our results suggest that CRS induced reductions in the number of $\mathrm{GFAP}^{+}$or $\mathrm{S} 100 \beta^{+}$ astrocytes may be an important element underlying the etiology of stress-related neuropathologies. ER stress plays an important role in the development of CRS related 
astrocytic functions [11]. Apoptosis is a highly regulated process to induce apoptotic cell death. Increased expression of ER stress markers (GRP78, CHOP and caspase12) have been reported to activate apoptosis, as they are critical mediators during ER stress-mediated apoptosis [32, 33]. Thus we also studied the changes of ER stress and apoptosis. We found that CRS could increase the expression of GRP78, CHOP and caspase 12, indicating that CRS could induce ER stress. Moreover, CRS increased apoptosis in the amygdala. The glucocorticoid receptor antagonist RU486 significantly inhibited the expression of GRP78, CHOP and caspase12, and reduced apoptosis, indicating that RU486 inhibits ER stress and subsequent ER stress-related apoptosis in the amygdala.

In conclusion, our study suggests that exposure to chronic restraint stress probably decreases astrocyte numbers and induces apoptosis and ER stress in the amygdala. This study presents the first in-vivo evidence that exposure to CRS triggers astroglial loss in the amygdala. RU486 significantly ameliorates abnormal behaviors in CRS-induced anxiety model rats. The protective effects of RU486 could be attributed to attenuation of ER stress, inhibition of apoptosis, astrocyte proliferation. These results show that stress-related mental illnesses, such as anxiety, which have previously been shown to affect neuronal plasticity, are also associated with glial injuries. Further investigations are required to determine whether astrocyte apoptosis is involved in these changes and to provide a comprehensive molecular picture of the temporal responses of stress-related brain diseases.

\section{Acknowledgments}

This work was Supported by the National Science Foundation Programs (N0.81430047) and the Hebei Graduate Student Innovation Fund Programs.

\section{Disclosure Statement}

None.

\section{Reference}

1 Tsigos C, Chrousos GP: Hypothalamic-pituitary-adrenal axis, neuroendocrine factors and stress. J Psychosom Res 2002;53:865-871.

2 McEwen BS, Gianaros PJ: Central role of the brain in stress and adaptation: links to socioeconomic status, health, and disease. Ann N Y Acad Sci 2010;1186:190-222.

3 Juruena MF, Cleare AJ, Pariante CM: [The hypothalamic pituitary adrenal axis, glucocorticoid receptor function and relevance to depression]. Rev Bras Psiquiatr 2004;26:189-201.

4 Padival MA, Blume SR, Rosenkranz JA: Repeated restraint stress exerts different impact on structure of neurons in the lateral and basal nuclei of the amygdala. Neuroscience 2013;246:230-242.

-5 Grillo CA, Risher M, Macht VA, Bumgardner AL, Hang A, Gabriel C, Mocaer E, Piroli GG, Fadel JR, Reagan LP: Repeated restraint stress-induced atrophy of glutamatergic pyramidal neurons and decreases in glutamatergic efflux in the rat amygdala are prevented by the antidepressant agomelatine. Neuroscience 2015;284:430-443.

6 Vassilopoulou K, Papathanasiou M, Michopoulos I, Boufidou F, Oulis P, Kelekis N, Rizos E, Nikolaou C, Pantelis C, Velakoulis D, Lykouras L: A magnetic resonance imaging study of hippocampal, amygdala and subgenual prefrontal cortex volumes in major depression subtypes: melancholic versus psychotic depression. J Affect Disord 2013;146:197-204.

7 Bowley MP, Drevets WC, Ongur D, Price JL: Low glial numbers in the amygdala in major depressive disorder. Biol Psychiatry 2002;52:404-412.

8 Herculano-Houzel S: The glia/neuron ratio: how it varies uniformly across brain structures and species and what that means for brain physiology and evolution. Glia 2014;62:1377-1391. 


\section{Cellular Physiology Cell Physiol Biochem 2017;42:1098-1108 \begin{tabular}{l|l} 
DOI: 10.1159/000478764 & $\begin{array}{l}\text { O 2017 The Author(s). Published by S. Karger AG, Basel } \\
\text { www.karger.com/cpb }\end{array}$ \\
\hline
\end{tabular} \\ Dong et al.: RU486 and Chronic Restraint Stress}

-9 Wang Q Verweij EW, Krugers HJ, Joels M, Swaab DF, Lucassen PJ: Distribution of the glucocorticoid receptor in the human amygdala; changes in mood disorder patients. Brain Struct Funct 2014;219:1615-1626.

10 Chen X, Lu M, He X, Ma L, Birnbaumer L, Liao Y: TRPC3/6/7 Knockdown Protects the Brain from Cerebral Ischemia Injury via Astrocyte Apoptosis Inhibition and Effects on NF-small ka, CyrillicB Translocation. Mol Neurobiol 2016;10.1007/s12035-016-0227-2

11 Shah A, Kumar A: Methamphetamine-mediated endoplasmic reticulum (ER) stress induces type-1 programmed cell death in astrocytes via ATF6, IRE1alpha and PERK pathways. Oncotarget 2016;7:4610046119.

12 Ji J, Zeng XN, Cao LL, Zhang L, Zhao Z, Yang DD, Sun XL: PPARbeta/delta activation protects against corticosterone-induced ER stress in astrocytes by inhibiting the CpG hypermethylation of microRNA-181a. Neuropharmacology 2017;113:396-406.

13 Wulsin AC, Herman JP, Solomon MB: Mifepristone decreases depression-like behavior and modulates neuroendocrine and central hypothalamic-pituitary-adrenocortical axis responsiveness to stress. Psychoneuroendocrinology 2010;35:1100-1112.

14 Wang SX, Chen JX, Yue GX, Bai MH, Kou MJ, Jin ZY: Xiaoyaosan decoction regulates changes in neuropeptide $\mathrm{y}$ and leptin receptor in the rat arcuate nucleus after chronic immobilization stress. Evid Based Complement Alternat Med 2012;2012:381278.

15 Zuloaga DG, Carbone DL, Hiroi R, Chong DL, Handa RJ: Dexamethasone induces apoptosis in the developing rat amygdala in an age-, region-, and sex-specific manner. Neuroscience 2011;199:535-547.

16 Brummelte S, Galea LA: Chronic high corticosterone reduces neurogenesis in the dentate gyrus of adult male and female rats. Neuroscience 2010;168:680-690.

17 Park HJ, Lee S, Jung JW, Kim BC, Ryu JH, Kim DH: Glucocorticoid- and long-term stress-induced aberrant synaptic plasticity are mediated by activation of the glucocorticoid receptor. Arch Pharm Res 2015;38:1204-1212.

18 Finsterwald C, Alberini CM: Stress and glucocorticoid receptor-dependent mechanisms in long-term memory: from adaptive responses to psychopathologies. Neurobiol Learn Mem 2014;112:17-29.

19 Wu TC, Chen HT, Chang HY, Yang CY, Hsiao MC, Cheng ML, Chen JC: Mineralocorticoid receptor antagonist spironolactone prevents chronic corticosterone induced depression-like behavior. Psychoneuroendocrinology 2013;38:871-883.

20 Solomon MB, Wulsin AC, Rice T, Wick D, Myers B, McKlveen J, Flak JN, Ulrich-Lai Y, Herman JP: The selective glucocorticoid receptor antagonist CORT 108297 decreases neuroendocrine stress responses and immobility in the forced swim test. Horm Behav 2014;65:363-371.

21 DeBattista C, Belanoff J, Glass S, Khan A, Horne RL, Blasey C, Carpenter LL, Alva G: Mifepristone versus placebo in the treatment of psychosis in patients with psychotic major depression. Biol Psychiatry 2006;60:1343-1349.

22 Li CL, Chen DJ, Song LP, Wang Y, Zhang ZF, Liu MX, Chen WL: Effectiveness and Safety of Lower Doses of Mifepristone Combined With Misoprostol for the Termination of Ultra-Early Pregnancy: A Dose-Ranging Randomized Controlled Trial. Reprod Sci 2015;22:706-711.

23 Liu C, Lu Q Qu H, Geng L, Bian M, Huang M, Wang H, Zhang Y, Wen Z, Zheng S, Zhang Z: Different dosages of mifepristone versus enantone to treat uterine fibroids: A multicenter randomized controlled trial. Medicine (Baltimore) 2017;96:e6124.

24 Kimelberg HK: Functions of mature mammalian astrocytes: a current view. Neuroscientist 2010;16:79-106.

-25 Musholt K, Cirillo G, Cavaliere C, Rosaria Bianco M, Bock J, Helmeke C, Braun K, Papa M: Neonatal separation stress reduces glial fibrillary acidic protein- and \$100beta-immunoreactive astrocytes in the rat medial precentral cortex. Dev Neurobiol 2009;69:203-211.

26 Ruvalcaba-Delgadillo Y, Luquin S, Ramos-Zuniga R, Feria-Velasco A, Gonzalez-Castaneda RE, Perez-Vega MI, Jauregui-Huerta F, Garcia-Estrada J: Early-life exposure to noise reduces mPFC astrocyte numbers and T-maze alternation/discrimination task performance in adult male rats. Noise Health 2015;17:216-226.

27 Bohn MC, Howard E, Vielkind U, Krozowski Z: Glial cells express both mineralocorticoid and glucocorticoid receptors. J Steroid Biochem Mol Biol 1991;40:105-111.

28 Tynan RJ, Beynon SB, Hinwood M, Johnson SJ, Nilsson M, Woods JJ, Walker FR: Chronic stress-induced disruption of the astrocyte network is driven by structural atrophy and not loss of astrocytes. Acta Neuropathol 2013;126:75-91. 
Cellular Physiology Cell Physiol Biochem 2017;42:1098-1108

\begin{tabular}{l|l} 
and Biochemistry Published 10.1159/000478764 & $\begin{array}{l}\text { (c) } 2017 \text { The Author(s). Published by S. Karger AG, Basel } \\
\text { www.karger.com/cpb }\end{array}$
\end{tabular}

Dong et al.: RU486 and Chronic Restraint Stress

29 Yu S, Yang S, Holsboer F, Sousa N, Almeida OF: Glucocorticoid regulation of astrocytic fate and function. PLoS One 2011;6:e22419.

-30 Salm AK: Mechanisms of glial retraction in the hypothalamo-neurohypophysial system of the rat. Exp Physiol 2000;85 Spec No:197S-202S.

-31 Bender CL, Calfa GD, Molina VA: Astrocyte plasticity induced by emotional stress: A new partner in psychiatric physiopathology? Prog Neuropsychopharmacol Biol Psychiatry 2016;65:68-77.

-32 Fulda S, Gorman AM, Hori O, Samali A: Cellular stress responses: cell survival and cell death. Int J Cell Biol 2010;2010:214074.

33 Bhat TA, Chaudhary AK, Kumar S, O'Malley J, Inigo JR, Kumar R, Yadav N, Chandra D: Endoplasmic reticulum-mediated unfolded protein response and mitochondrial apoptosis in cancer. Biochim Biophys Acta 2017;1867:58-66. 